\title{
The Application of Autogenous Bone for the Reconstruction of Traumatic Defect of Orbital Floor
}

\author{
Chunlei Zhang', Ziyong Zhang ${ }^{2, *}$ \\ ${ }^{1}$ Department of Stomatology, The First Affiliated Hospital of Jinan University, Guangzhou, China \\ ${ }^{2}$ College of Life Science and Technology, Jinan University, Guangzhou, China
}

Email address:

chunlei_zhang@yeah.net (Ziyong Zhang)

${ }^{*}$ Corresponding author

\section{To cite this article:}

Chunlei Zhang, Ziyong Zhang. The Application of Autogenous Bone for the Reconstruction of Traumatic Defect of Orbital Floor. International Journal of Dental Medicine. Vol. 3, No. 4, 2017, pp. 16-19. doi: 10.11648/j.ijdm.20170304.11

Received: November 3, 2017; Accepted: December 11, 2017; Published: January 8, 2018

\begin{abstract}
To study the effects of reconstruction of orbital floor defects with autogenous bone, 20 patients with orbital floor fractures were treated via peri-orbital mini-incision, and orbital floor defects were reconstructed with autogenous bone. The results showed that 20 cases recovered well. There was no infection, optic neuropathy, diplopia and enophthalmos. In general, the data of the study suggest that collagen membrane is an ideal material for orbital reconstruction.
\end{abstract}

Keywords: Orbital Floor Fracture, Orbital Floor Reconstruction, Enophthalmos, Autogenous Bone

\section{Instruction}

Traumatic defect of orbital floor would lead to facial deformity and dysfunction, including diplopia, eye retraction, eye movement disorders and et al. Further, zygomatic complex fractures, maxillary fractures and nasal orbital sieve composite fractures are often accompanied with orbital fracture. Once this area is damaged, injuries of patients become complex, making it one of the difficulties in the treatment of facial fractures [1]. Since 2013, there were 20 cases of zygomatic complex fractures accompanied with orbital bone defects in our department. By the reconstruction of orbital bone with autogenous bone (maxillary sinus anterior wall and iliac bone piece), we significantly corrected the deformity and dysfunction, resulted in satisfied clinical effects.

\section{Method}

\subsection{Clinical Data Collection}

Since Jan 2013, patients with zygomatic complex with orbital bone defect from the department of Stomatology in the First Affiliated Hospital of Jinan University were included in the study. Inclusion criteria: (1) with symptoms of visual acuity, eye movement and location abnormalities and infraorbital nerve numbness; (2) CT scanning showed orbital floor fractures or orbital hernia went into the maxillary sinus; (3) can be reviewed six months later. There were 20 cases applied orbital floor reconstruction: 18 male and 2 females; aged from 19 to 42, average 27; all cases showed infraorbital margin; 13 cases showed lateral eye and neurological dysfunction; trauma time ranged from 3 days to 1 month; cause of trauma, 14 cases from traffic accident, 5 from fight and 1 from fall.

\subsection{Examination}

Record the preoperative data of facial deformity, eye position and function, occlusion situation, CT images, size of fracture, facial scar and nerve function. Patients were followed up six months later, postoperative data were collected and compared with preoperative data to evaluate the clinical effects.

\subsection{Operational Methods}

Conventional anesthesia surgery, small facial incisions were applied: eyelid margin, eyebrow combined with mouth gingival sulcus incision. When performing the zygomatic complex fracture surgery, the reconstructions of orbital bone defect were also performed. In patients with orbital floor probe, different parts of autogenous bones were selected 
according to the serious situation of zygomatic fracture and orbital floor fracture. For those with light zygomatic fractures shift and fracture $<1.5 \mathrm{~cm}$, maxillary sinus anterior bone wall was used for the reconstruction; for those with eye movement, visual and infraorbital nerve dysfunction, with fracture $>=1.5$ $\mathrm{cm}$, decompression was applied for extraocular muscles and infraorbital nerve, falling orbital soft tissues were accurately relocated. Iliac bone pieces were used for the reconstruction. With titanium plate and micro-nail for the fixation, layered suture, small films were placed for drainage. General antibiotic treatment, stitches were removed 5-7 days after the surgery.

\subsection{Statistical Analysis}

The data were analyzed by SPSS 20.0, paired t-test for

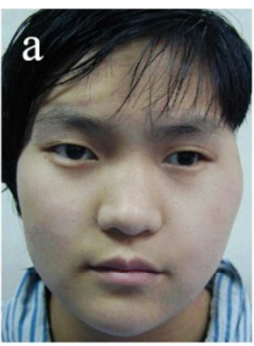

preoperation

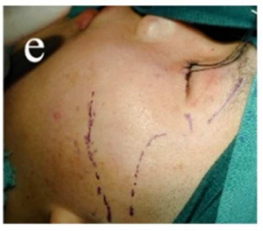

preoperative line

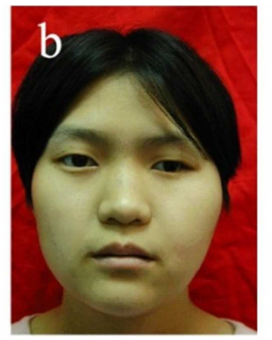

6 months postoperation

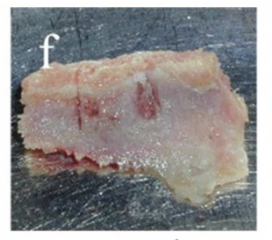

autogenous bone

statistical analysis, $\mathrm{p}<0.05$ showed significant differences.

\section{Results}

Postoperative follow-up was applied for the long-term observation of the surgical effects. Six month later, the data of facial deformity, eye position and function, occlusion situation, CT images, size of fracture, facial scar and nerve function were recorded and compared with preoperative data. The evaluation standard is shown in Table 1. 80\% (16/20) of patients were recovered, $20 \%(4 / 20)$ of patients improved, 0 cases showed invalid. No complications were found because of orbital floor bone reconstruction. The prognosis data were shown in Table 2. Representative cases were shown in Figure 1.

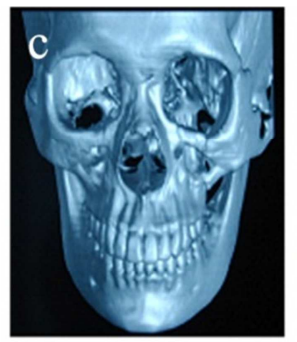

preoperative $\mathrm{CT}$

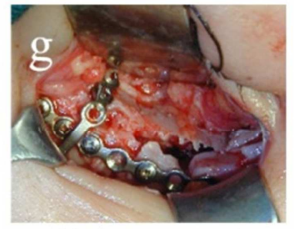

orbital reconstruction

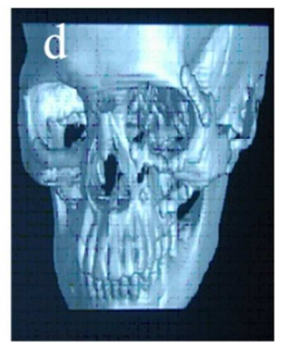

postoperative CT

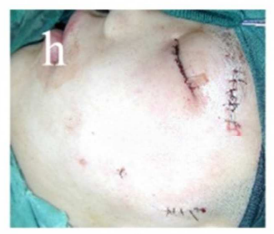

postoperative suture

Figure 1. Presentative case.

Table 1. The evaluation standard for clinical effect.

\begin{tabular}{llll}
\hline Effects & Facial appearance & Eye position and function & Occlusion \\
\hline \multirow{3}{*}{ Recovered } & \multirow{2}{*}{ Symmetry } & Normal appearance & Normal \\
& & Normal pupil horizontal position & \\
& & Normal eye movement & \\
Improved & Basically normal & Pupil horizontal position down & Slightly wrong occlusive deformity \\
& & Restricted eye movement & \\
Invalid & asymmetry & Eye subsidence & Obvil horizontal position down \\
& & Eye movement is limited & \\
\hline
\end{tabular}

Table 1. Continued.

\begin{tabular}{|c|c|c|c|c|}
\hline Effects & Imaging evaluation & Opening degree & Scar & Facial paralysis \\
\hline Recovered & $\begin{array}{l}\text { Fracture line alignment } \\
\text { Completely healed bone ends } \\
\text { No bone absorption } \\
\text { Normal shape }\end{array}$ & Normal & No & No \\
\hline Improved & $\begin{array}{l}\text { Fracture line alignment slightly worse } \\
\text { Bone fracture healing slightly worse } \\
\text { Have a slight bone resorption } \\
\text { The shape is normal }\end{array}$ & Slightly restricted & Not obvious & Not obvious \\
\hline Invalid & $\begin{array}{l}\text { Fracture line alignment } \\
\text { Bad bone fracture healing } \\
\text { Bone absorption more obvious } \\
\text { Asymmetric shape }\end{array}$ & Severely limited & Obvious & Yes \\
\hline
\end{tabular}


Table 2. The prognosis data.

\begin{tabular}{lll}
\hline Effects & Cases & Percentage(\%) \\
\hline Recovered & 16 & 80 \\
Improved & 4 & 20 \\
Invalid & 0 & 0 \\
In total & 20 & 100 \\
\hline
\end{tabular}

\section{Discussion}

Complex zygomatic fractures combined with orbital floor defect often induces the content of hernia go into ethmoid or maxillary sinus, making the eye ball move down and finally leading to diplopia $[2,3]$. Current ideal surgeries to reconstruct must fulfill the following requirement: (1) restore the integrity of the orbital bone structure; (2) reset the orbital contents, release eye muscle incarceration, correct diplopia and eye retraction. Thus, to correctly calculate the size, shape and position of the defect becomes the most difficult problem. Researchers [4] indicated that each $1 \mathrm{~cm}$ increase of orbital contents, eye prominence would move forward 0.47-1.00 mm, which cannot be clinically referenced. The clinical treatment currently relies mainly on eye judgement, making the effect of large discrepancy. Materials for reconstruction include two types: autogenous and non-biological materials [5-7]. The commonly used autografts include: iliac, skull, costal cartilage, nasal septum cartilage and so on. And the non-biological materials including: titanium mesh, PLLA, PGA, teflon, polyethylene, hydroxyapatite and so on. Non-biological materials are easy to shape and able to provide solid support, the surgical time is short, but often accompanied with disadvantages of implanting displacement and infection. Medical silicone also is easy to reform, but with not enough hardness and are easy to deform or shift off. Polyethylene has good biocompatibility, toughness and stability, easier to shape and to obtain accurate three-dimensional shape [8]. Aitasalo and colleagues [9] used bio-active glass for the reconstruction of orbital defects, considering its antibacterial activity and biological activity, but hard to shape and hard to absorb. Absorbable polymers (PGA, PLLA, PDS) can be used for orbital defect reconstruction, no antigenicity, but the degradation time is longer, foreign body reaction may appear and the mechanical strength would decrease with the degradation. Titanium is widely used for the reconstruction, with extremely stable physical and chemical property, good biological compatibility and can be long-term placed in human body, however, this material may not be good for the development of adolescent. In general, to select the appropriate material for the reconstruction is a college work for surgeons [4].

Recent years, more and more doctors use autogenous bones for the orbital reconstruction. And we got satisfied effect in the study using autogenous bones. Kronig and colleagues $[10,11]$ suggested that using autologous bones to construct the orbital floor can correct enophthalmos and acquire excellent function and aesthetic shape, can decrease the enlarged orbital volume, remove ocular subsidence deformity. Autografts shown no exclusion, and directly fit the orbital structure. However, there are also some disadvantages of using autogenous bones: need secondary surgical field, hard to shape and increased secondary drama [12-14]. Currently, quantitative surgical designing system under the guidance of medical 3D imaging and rapid 3D printing prototyping technique has been deployed [15], trying to explore the best treatment for the complex orbital floor fractures and to fix the difficulties of maxillofacial deformity, enophalmos, eye subsidence and other problems.

\section{Conflict of Interest}

The authors declare no conflict of interest.

\section{References}

[1] D. Bartoli, M. T. Fadda, A. Battisti, A. Cassoni, M. Pagnoni, E. Riccardi, M. Sanzi, V. Valentini, Retrospective analysis of 301 patients with orbital floor fracture, J Craniomaxillofac Surg, 43 (2015) 244-247.

[2] P. N. Manson, A. Grivas, A. Rosenbaum, M. Vannier, J. Zinreich, N. Iliff, Studies on enophthalmos: II. The measurement of orbital injuries and their treatment by quantitative computed tomography, Plast Reconstr Surg, 77 (1986) 203-214.

[3] E. Zunz, O. Blanc, I. Leibovitch, Traumatic orbital floor fractures: repair with autogenous bone grafts in a tertiary trauma center, J Oral Maxillofac Surg, 70 (2012) 584-592.

[4] R. W. Whitehouse, M. Batterbury, A. Jackson, J. L. Noble, Prediction of enophthalmos by computed tomography after 'blow out' orbital fracture, Br J Ophthalmol, 78 (1994) 618-620.

[5] M. Totir, R. Ciuluvica, I. Dinu, I. Careba, S. Gradinaru, Biomaterials for orbital fractures repair, J Med Life, 7 Spec No. 4 (2014) 62-64.

[6] D. R. Gunarajah, N. Samman, Biomaterials for repair of orbital floor blowout fractures: a systematic review, J Oral Maxillofac Surg, 71 (2013) 550-570.

[7] J. K. Potter, M. Malmquist, E. Ellis, 3rd, Biomaterials for reconstruction of the internal orbit, Oral Maxillofac Surg Clin North Am, 24 (2012) 609-627.

[8] J. Lin, M. German, B. Wong, Use of copolymer polylactic and polyglycolic acid resorbable plates in repair of orbital floor fractures, Facial Plast Surg, 30 (2014) 581-586.

[9] K. Aitasalo, I. Kinnunen, J. Palmgren, M. Varpula, Repair of orbital floor fractures with bioactive glass implants, J Oral Maxillofac Surg, 59 (2001) 1390-1395; discussion 1395-1396.

[10] V. Garg, G. B. Giraddi, S. Roy, Comparison of efficacy of mandible and iliac bone as autogenous bone graft for orbital floor reconstruction, J Maxillofac Oral Surg, 14 (2015) 291-298.

[11] S. A. Kronig, R. J. van der Mooren, E. M. Strabbing, L. H. Stam, J. A. Tan, E. de Jongh, K. G. van der Wal, D. Paridaens, M. J. Koudstaal, Pure orbital blowout fractures reconstructed with autogenous bone grafts: functional and aesthetic outcomes, Int J Oral Maxillofac Surg, 45 (2016) 507-512. 
[12] C. R. Bande, S. Daware, P. Lambade, B. Patle, Reconstruction of Orbital Floor Fractures with Autogenous Bone Graft Application from Anterior Wall of Maxillary Sinus: A Retrospective Study, J Maxillofac Oral Surg, 14 (2015) 605-610.

[13] J. E. O'Connell, C. Hartnett, M. Hickey-Dwyer, G. J. Kearns, Reconstruction of orbital floor blow-out fractures with autogenous iliac crest bone: a retrospective study including maxillofacial and ophthalmology perspectives, J Craniomaxillofac Surg, 43 (2015) 192-198.
[14] J. Al-Sukhun, H. Penttila, N. Ashammakhi, Orbital stress analysis: part II: design and fixation of autogenous bone graft used to repair orbital blowout fracture, J Craniofac Surg, 22 (2011) 1294-1298.

[15] S. Z. Tabakovic, V. S. Konstantinovic, R. Radosavljevic, D. Movrin, M. Hadzistevic, N. Hatab, Application of Computer-Aided Designing and Rapid Prototyping Technologies in Reconstruction of Blowout Fractures of the Orbital Floor, J Craniofac Surg, 26 (2015) 1558-1563. 